\title{
0-GIcNAc transferase suppresses necroptosis and liver fibrosis
}

\author{
Bichen Zhang, ${ }^{1}$ Min-Dian Li, ${ }^{1}$ Ruonan Yin, ${ }^{2}$ Yuyang Liu, ${ }^{3}$ Yunfan Yang, ${ }^{4}$ Kisha A. Mitchell-Richards, ${ }^{5}$ \\ Jin Hyun Nam, ${ }^{6}$ Rui Li, ${ }^{7}$ Li Wang, ${ }^{8,9}$ Yasuko Iwakiri, ${ }^{10}$ Dongjun Chung, ${ }^{6}$ Marie E. Robert, ${ }^{11}$ \\ Barbara E. Ehrlich, ${ }^{1,12}$ Anton M. Bennett, ${ }^{4,12}$ Jun Yu, ${ }^{7}$ Michael H. Nathanson, ${ }^{10}$ and Xiaoyong Yang ${ }^{1,4}$ \\ 'Department of Cellular and Molecular Physiology and ²Department of Cell Biology, Yale University School of Medicine, \\ New Haven, Connecticut, USA. ${ }^{3}$ Yale College, Yale University, New Haven, Connecticut, USA. ${ }^{4}$ Program in Integrative Cell \\ Signaling and Neurobiology of Metabolism, Department of Comparative Medicine, Yale University School of Medicine, \\ New Haven, Connecticut, USA. ${ }^{5}$ Greenwich Hospital, Greenwich, Connecticut, USA. ${ }^{6}$ Department of Public Health Sciences, \\ Medical University of South Carolina, Charleston, South Carolina, USA. ${ }^{7}$ Institute of Digestive Disease and Department \\ of Medicine and Therapeutics, State Key Laboratory of Digestive Disease, Li Ka Shing Institute of Health Sciences, \\ CUHK Shenzhen Research Institute, Chinese University of Hong Kong, Hong Kong, China. ${ }^{8}$ Department of Physiology \\ and Neurobiology and ${ }^{9}$ Institute for Systems Genomics, University of Connecticut, Storrs, Connecticut, USA. ${ }^{10}$ Section of \\ Digestive Diseases, Department of Internal Medicine, ${ }^{11}$ Department of Pathology, and ${ }^{12}$ Department of Pharmacology, Yale \\ University School of Medicine, New Haven, Connecticut, USA
}

Worldwide, over a billion people suffer from chronic liver diseases, which often lead to fibrosis and then cirrhosis. Treatments for fibrosis remain experimental, in part because no unifying mechanism has been identified that initiates liver fibrosis. Necroptosis has been implicated in multiple liver diseases. Here, we report that $\mathrm{O}$-linked $\beta$ - $\mathrm{N}$-acetylglucosamine (O-GIcNAc) modification protects against hepatocyte necroptosis and initiation of liver fibrosis. Decreased 0-GIcNAc levels were seen in patients with alcoholic liver cirrhosis and in mice with ethanol-induced liver injury. Liver-specific 0-GIcNAc transferase-KO (OCT-LKO) mice exhibited hepatomegaly and ballooning degeneration at an early age and progressed to liver fibrosis and portal inflammation by 10 weeks of age. OCT-deficient hepatocytes underwent excessive necroptosis and exhibited elevated protein expression levels of receptor-interacting protein kinase 3 (RIPK3) and mixed lineage kinase domain-like (MLKL), which are key mediators of necroptosis. Furthermore, glycosylation of RIPK3 by OCT is associated with reduced RIPK3 protein stability. Taken together, these findings identify OCT as a key suppressor of hepatocyte necroptosis, and OCT-LKO mice may serve as an effective spontaneous genetic model of liver fibrosis.

Conflict of interest: The authors have declared that no conflict of interest exists.

Copyright: (c) 2019, American Society for Clinical Investigation.

Submitted: April 4, 2019 Accepted: September 27, 2019 Published: November 1, 2019

Reference information: $/ C I$ Insight 2019;4(21):e127709. https://doi.org/10.1172/jci. insight.127709.

\section{Introduction}

More than a billion people worldwide are afflicted by chronic liver disease, the most common causes of which include fatty liver, alcohol, and infection with hepatitis B or C virus. Despite differences in the etiology of these chronic liver diseases, they each lead to excessive stress on the liver and result in hepatocyte death (1). Hepatocellular death contributes significantly to chronic liver disease by inducing inflammatory responses that lead to hepatic fibrosis and cirrhosis (2). Recent evidence suggests that necroptosis, a form of caspase-independent, regulated cell death, is manifested in a variety of acute and chronic types of liver diseases $(3,4)$. Necroptosis has been implicated in patients with alcoholic steatohepatitis (ASH), nonalcoholic steatohepatitis (NASH), and hepatitis B or C infections $(5,6)$, underscoring its prevalence and general role in the pathogenesis of chronic liver diseases.

Necroptosis is mediated by the necrosome, a signaling protein complex that is composed of receptor-interacting protein kinase 3 (RIPK3) and mixed lineage kinase domain-like (MLKL) (7). Phosphorylated RIPK3 recruits and activates MLKL, which translocates to the cell membrane and causes membrane rupture (8). Owing to the loss of membrane integrity in necroptotic cells, the intracellular contents act as damage-associated molecular patterns (DAMPs) that recruit immune cells and cause sterile inflammation in affected tissues (9). Necroptosis also promotes cell-autonomous production of proinflammatory cytokines (10). 
Therefore, necroptosis is considered as a highly inflammatory form of cell death. In light of the data collected from patients, a myriad of studies in recent years have investigated the contribution of necroptosis in different mouse liver injury models and demonstrated the importance and complexity of necroptosis in these pathological conditions (11-16). However, the mechanism that governs necroptotic hepatocyte death is largely unknown.

O-GlcNAcylation is the attachment of O-GlcNAc modification on serine or threonine residues of cytoplasmic, nuclear, and mitochondrial proteins. It is a dynamic and reversible posttranslational modification (PTM) that regulates a broad spectrum of cellular events ranging from epigenetics and transcription to signaling and rhythm (17-20). By sensing and integrating the nutrient and stress cues, it prepares cells to respond promptly to an ever-changing microenvironment. A pair of enzymes, O-GlcNAc transferase (OGT) and O-GlcNAcase (OGA), actively controls the cycling of O-GlcNAc modification. Preserving the O-GlcNAc level in an optimal zone is essential for maintaining cellular homeostasis, whereas aberrant O-GlcNAcylation is linked to a plethora of diseases, including cancer, metabolic syndromes, and cardiovascular diseases (21-23). Throughout the years, it has become evident that dysfunction of O-GlcNAcylation sensitizes cells to various kinds of stress, ultimately leading to cell death $(24,25)$. This phenomenon has been repeatedly reported in various tissues and pathologies (26-29), yet the mechanisms by which the perturbation of O-GlcNAcylation affects cell survival and death are undefined.

Previously, we have shown that OGT performs essential functions in liver physiology by regulating gluconeogenesis and the insulin signaling pathway $(30,31)$, the circadian clock (32), and autophagy (33). Germline OGT deletion is lethal at the embryogenesis stage, and previous models studying hepatic OGT are based on transient genetic interventions. A major caveat is that we did not know the chronic effects of OGT deletion on hepatocytes. Here, we report that chronic hepatocyte-specific deletion of OGT leads to a spontaneous, rapidly developing liver injury due to massive hepatocellular necroptosis. We further demonstrate that OGT serves as a negative regulator of necroptosis by suppressing RIPK3 expression. These findings reveal that hepatocyte OGT protects the liver against necroptosis and, thus, liver fibrosis.

\section{Results}

Impaired O-GlcNAc signaling in humans and mice with liver injury. To examine the involvement of O-GlcNAc modification in chronic liver disease, we investigated the changes in O-GlcNAc signaling in patients with alcoholic liver cirrhosis. The expression of $\alpha$ smooth muscle actin $(\alpha \mathrm{SMA})$ was greatly increased in patients, indicating excessive extracellular matrix (ECM) deposition and liver injury in these patients (Figure 1, A and B). Global O-GlcNAc levels were decreased in the livers from cirrhosis patients, which was associated with decreased OGT protein level and increased OGA protein level (Figure 1, A and B). We also found a significant increase in the level of phosphorylated MLKL, indicating the augmentation of necroptosis in patients with alcoholic liver cirrhosis. These results show that both O-GlcNAc and necroptosis signaling are associated with the development of liver cirrhosis in patients. We also examined the changes in O-GlcNAc levels in a mouse model of ethanol-induced liver injury. Mice were fed with a Lieber-DeCarli liquid diet to induce alcoholic steatosis and liver inflammation (34). Decreased liver O-GlcNAc levels were seen in mice fed with the ethanol diet as compared with the control group, which could be explained by the reduced expression of OGT in ethanol-fed mice (Figure 1C). Phosphorylation of MLKL is also increased in these mice, which is in agreement with the previous report that necroptosis is induced in the mouse model of alcoholic liver disease (35). Taken together, these results indicate that decreased O-GlcNAcylation is correlated with the induction of necroptosis in humans and mice with chronic liver injury.

Loss of OGT in hepatocytes leads to rapidly developed hepatomegaly and ballooning degeneration in mice. To investigate the physiological roles of OGT in the liver, we generated liver-specific OGT-KO mice (OGT-LKO) and control littermates (WT) by crossing Albumin-Cre; Ogt $t^{F / Y}$ mice with $O g t^{F / F}$ mice. Both mRNA and protein levels of OGT were significantly reduced in OGT-LKO mouse livers, confirming $\mathrm{KO}$ efficiency (Figure 2A and Supplemental Figure 1A; supplemental material available online with this article; https://doi.org/10.1172/jci.insight.127709DS1). As a result of OGT deletion, global O-GlcNAc levels were also diminished in hepatocytes, as demonstrated by Western blot and IHC analyses (Figure 2A and Supplemental Figure 1B). WT and OGT-LKO mice had comparable body weight and showed no difference in oxygen consumption, food intake, and physical activities when measured at 4, 10, and 24 weeks of age (Supplemental Figure 2; data shown were measured at 10 weeks of age). However, at 4 weeks of age, OGT-LKO mice exhibited hepatomegaly and elevated circulating 
A
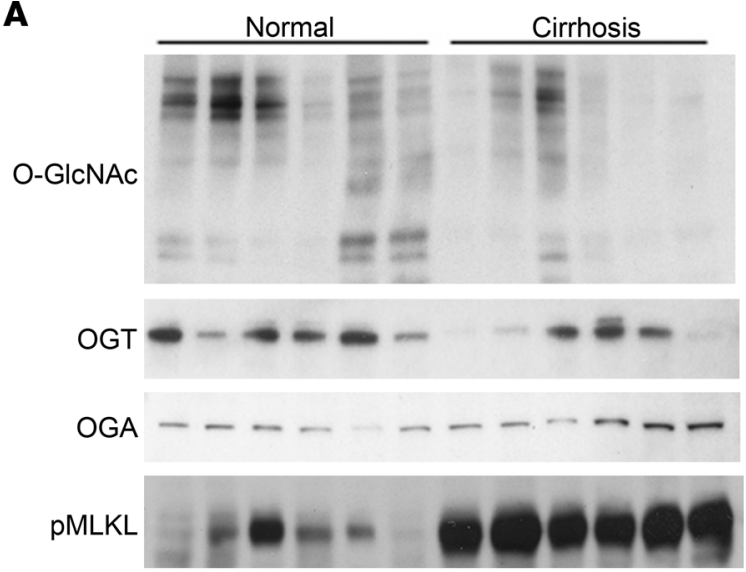

MLKL

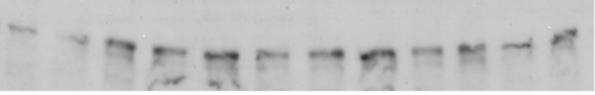

aSMA

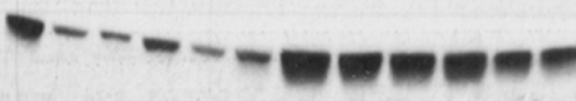

C

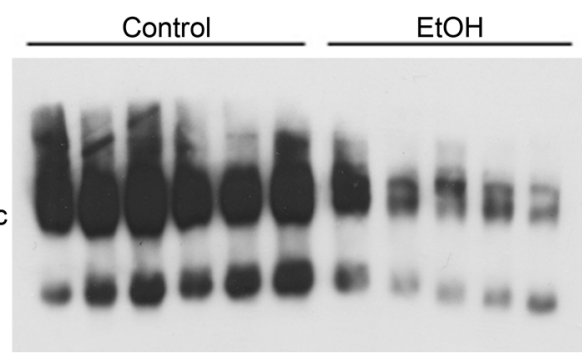

OGT
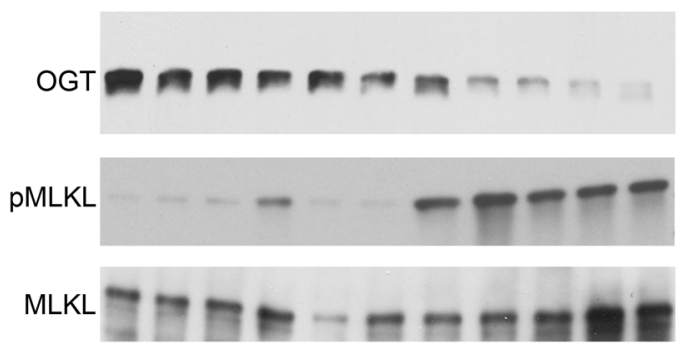

GAPDH

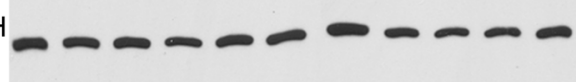

Ponceau S

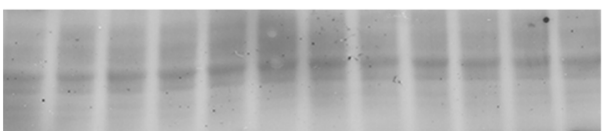

B

OGT
OGA
pMLKL
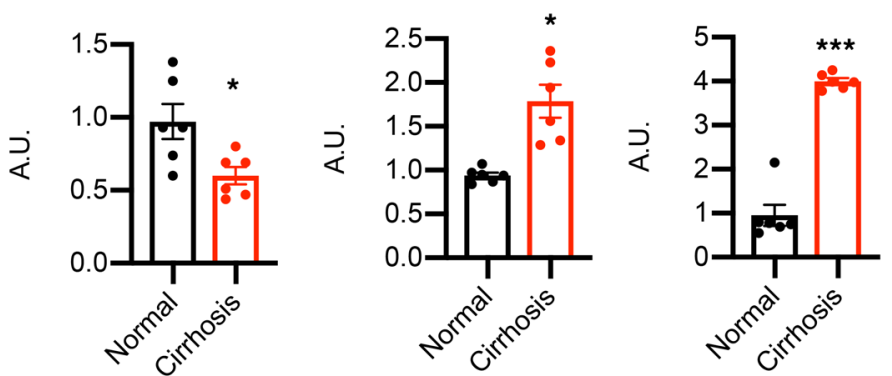

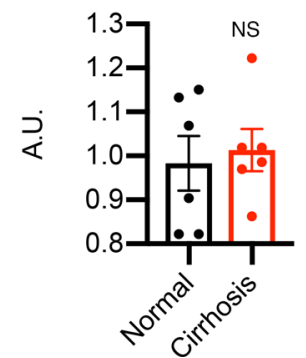

MLKL

aSMA

Figure 1. Defective 0-GIcNAc signaling in patients with liver cirrhosis and in mice with ethanol-induced liver injury. (A) Western blots showing the protein expression in livers from healthy control and cirrhosis patients ( $n=6$ for both groups). (B) Densitometric analysis of Western blot results shown in A. (C) Western blots showing the protein expression in livers from control or ethanol diet-fed mice. $n=6$ for control group and $n=5$ for ethanol diet-fed group. Data are shown as mean \pm SEM. ${ }^{*} P<0.05 ;{ }^{* *} P<0.001$ by unpaired Student's $t$ test. 0 -GIcNAc, 0 -linked $\beta$-N-acetylglucosamine.

alanine aminotransferase (ALT) and aspartate aminotransferase (AST) levels, indicating a rapid development of liver injury in these mice (Figure 2, B-E, and Supplemental Figure 1, C and D). This injury was not due to developmental defects, since no abnormality was identified in 1-week-old OGT-LKO mice (Supplemental Figure 1, E-G). We performed pathological staining and scoring to further analyze the changes in the KO mice. Histological analysis of H\&E-stained liver sections identified ballooning degeneration in the OGT-LKO liver, as shown by swollen hepatocytes, vacuolated cytoplasm, and accumulation of Mallory hyaline (Figure 2, F and G). Mild collagen deposition and sinusoidal fibrosis were observed in Masson's trichrome stains (Supplemental Figure 1H). Although the hydroxyproline content was not significantly higher in the OGT-LKO liver (Supplemental Figure 1I), pathology scores revealed that 4 -week-old $\mathrm{KO}$ mice were in early stages of liver fibrosis $(16.7 \%$ in stage 1a, $66.7 \%$ in stage $1 \mathrm{c}$, and $16.6 \%$ in stage 2), whereas all WT mice were healthy (Figure $2 \mathrm{H}$ ). Alongside the histological observations, the mRNA levels of fibrogenic genes (Acta2, Colla1, and Mmp2) were increased in 4-week-old OGT-LKO livers (Supplemental Figure 1J). Injured hepatocytes release cytokines that cause inflammation in the liver (36). We found the increased mRNA levels of proinflammatory genes $(C c l 2, I l 6$, and Tnfa) in OGT-LKO mice (Figure 2I). Moreover, proinflammatory cytokines TNFA and 
A

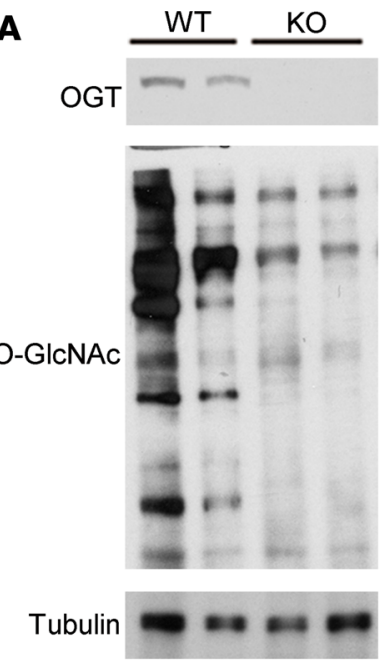

B

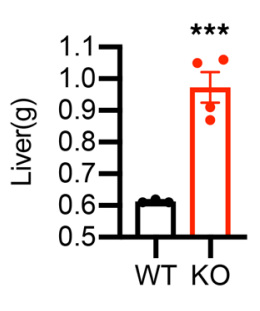

D

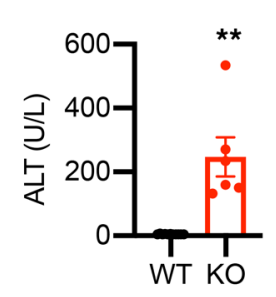

C

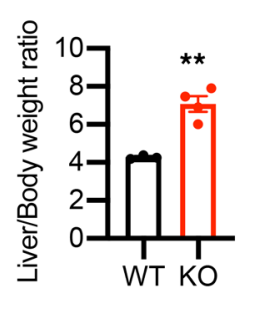

E

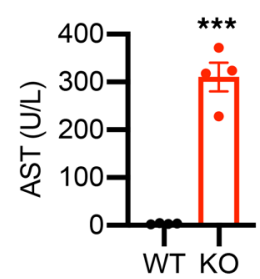

$\mathbf{F}$
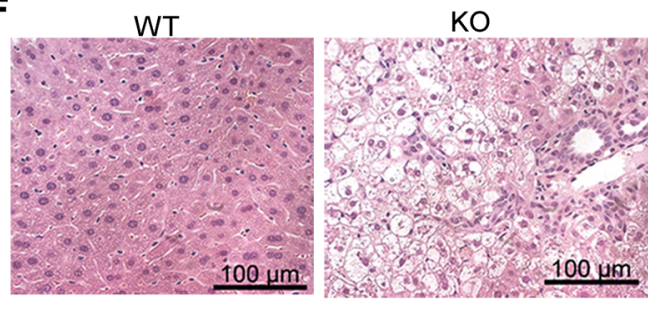

G

Ballooning degeneration Mallory hyaline

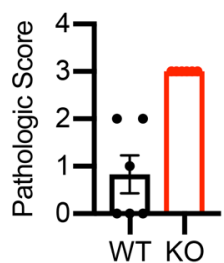

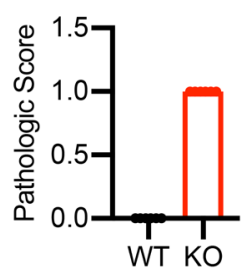

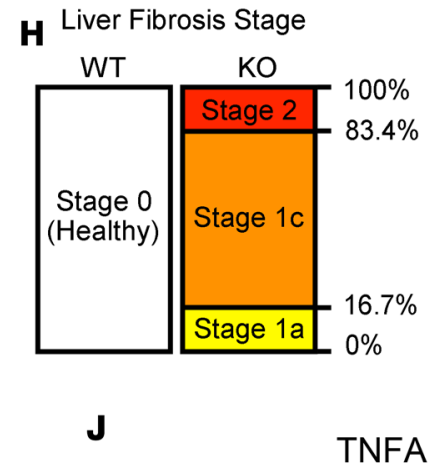

Liver

TNFA
I
$1 / 6$

Tnfa

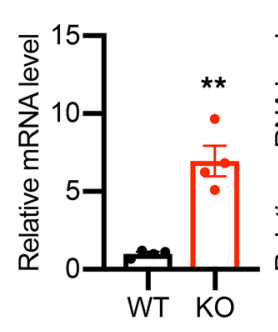

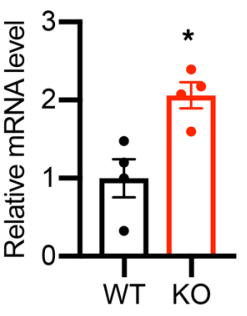

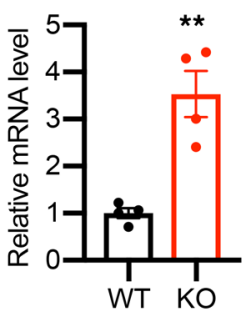

IL 6
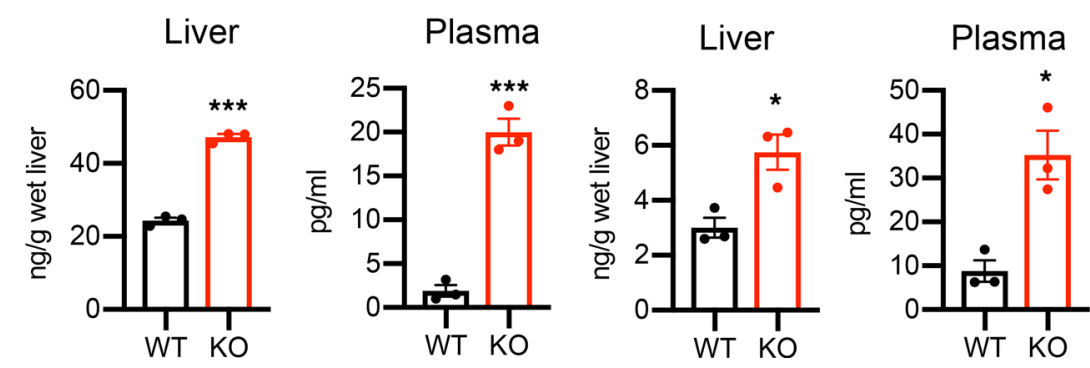

Figure 2. Hepatocyte-specific deletion of OGT leads to hepatomegaly and liver injury in 4-week-old KO mice. (A) Western blots showing the deletion of OGT and decrease of O-GIcNAc levels in OCT-LKO mouse livers. (B) Liver weight. (C) Percentage of liver weight to body weight. (D and E) ALT and AST levels in the plasma from WT and OCT-LKO mice. (F) H\&E stains showing the ballooning degeneration in OGT-LKO livers. (C) Pathologic scores of liver sections from WT and OGT-LKO mice. (H) Liver fibrosis stages in 4-week-old mice. (I) Expression levels of proinflammatory genes. (J) Liver and plasma TNFA and IL-6 levels determined by ELISA $(n=3) . n=4-6$, both sexes. Data are shown as mean \pm SEM. ${ }^{*} P<0.05 ;{ }^{* *} P<0.01 ;{ }^{* *} P<0.001$ by unpaired Student's $t$ test. 0 -GlcNAc, 0 -linked $\beta$-N-acetylglucosamine; OCT, O-GIcNAc transferase; OGT-LKO, liver-specific OGT knockout; ALT, alanine aminotransferase; AST, aspartate aminotransferase.

IL-6 were increased both in the liver and in the plasma of OGT-LKO mice (Figure 2J). Together, these results demonstrate that OGT-LKO mice develop spontaneous liver damage in 4 weeks.

OGT deletion in hepatocytes leads to global transcriptome changes in the liver. Given the liver injury observed in OGT-LKO mice at 4 weeks, we performed RNA sequencing (RNA-seq) to further analyze transcriptional changes in the OGT-LKO livers. The transcriptome analysis of the liver from OGT-LKO mice revealed profound changes in gene expression patterns as compared with WT mice (Figure 3A and Supplemental Figure 3A). A total of 2,341 genes showed at least 2-fold changes in expression between WT and OGT-LKO mice, among which 1,525 genes were upregulated and 816 genes were downregulated (Figure 3B, Supplemental Figure 3B, and Supplemental Table 1). In agreement with our observation of early-onset fibrosis in 4-weekold OGT-LKO mice, gene set enrichment analysis revealed that the ECM-associated pathway was among the 


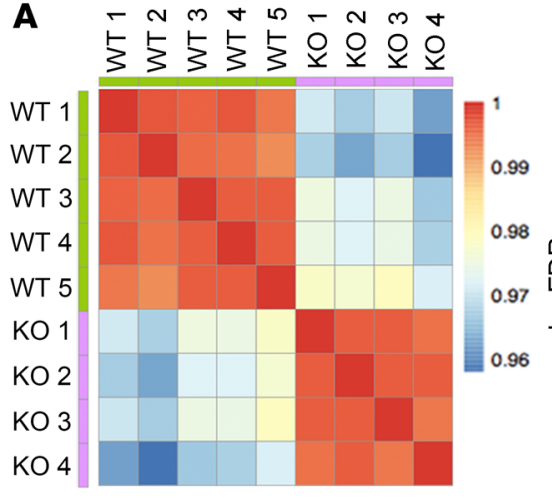

C

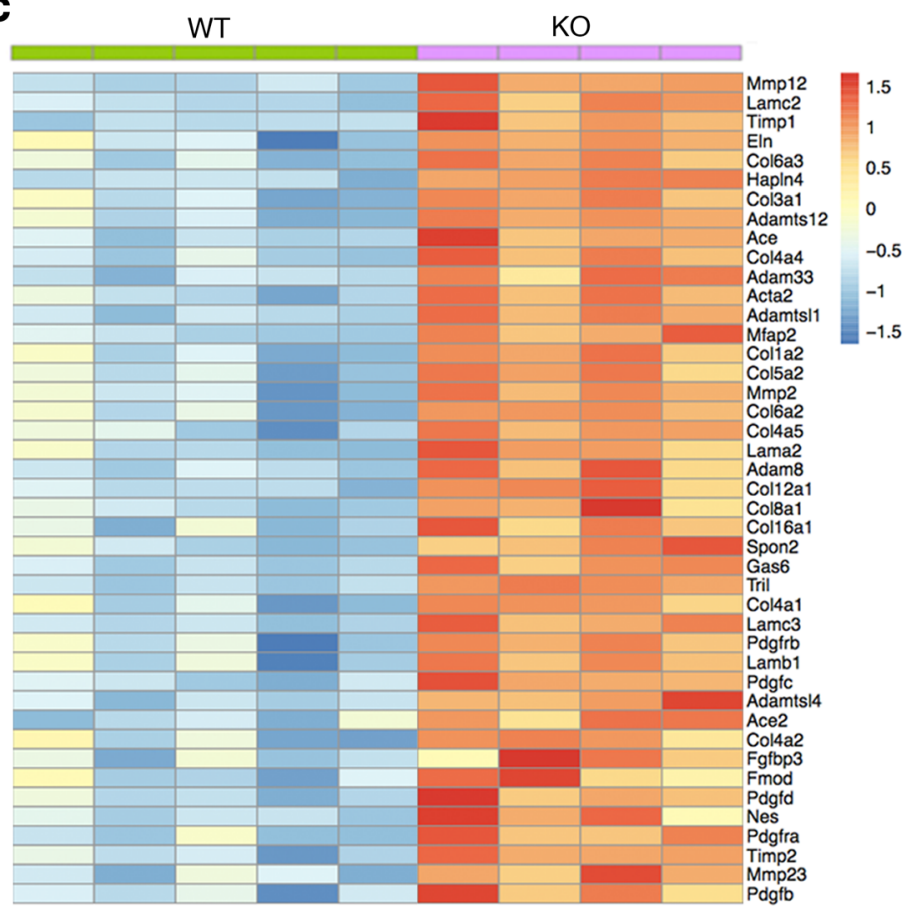

$\begin{array}{lll}\text { B Down: } 816 \text { genes Up: } 1525 \text { genes } & \end{array}$

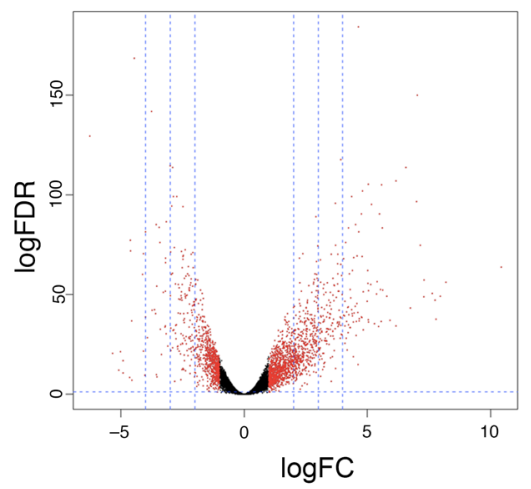

Figure 3. RNA-sequencing analysis reveals global transcriptional changes in OGT-LKO mouse livers at 5 weeks of age. (A) Heatmap of correlation matrix. (B) Volcano graph showing up- and downregulation of genes. (C) Representative genes in the ECM organization pathway that are upregulated in OCT-LKO livers. OCT-LKO, liver-specific OCT KO; ECM, extracellular matrix.

most enriched pathways in KO livers (Table 1). Consistently, the mRNA expression levels of key mediators in ECM organization and collagen formation were elevated in OGT-LKO mice (Figure 3C). In contrast, genes that encode enzymes involved in amino acid or lipid metabolism, xenobiotic metabolism, and detoxification were greatly repressed in OGT-LKO mice, indicating the impairment of major functions in hepatocytes from KO mice (Table 2). Differentially expressed genes were also investigated with mouse phenotype enrichment analysis and gene-disease association analysis. Top results in these analyses were abnormal response to injury (Mouse Phenotype ID: 0005164) and experimental liver cirrhosis (C0023893, DisGeNET). These results indicate that OGT-LKO mice quickly develop the fibrogenic program with global transcriptome changes and potentially serve as a genetic model by which to study liver fibrosis.

OGT-LKO mice progress to liver fibrosis and portal inflammation at 10 weeks of age. Disruption of normal hepatocellular architecture and elevated expression of proinflammatory genes indicate the possibility of progression to liver fibrosis, which is a critical phase in the development of human chronic liver disease. We monitored the progression of liver injury in OGT-LKO mice and found that 10 -week-old OGT-LKO mice exhibited more severe hepatomegaly with higher ALT and AST levels as compared with WT mice (Figure 4A and Supplemental Figure 4A). The H\&E staining of OGT-LKO mouse liver sections showed massive immune cell infiltration that indicated portal and lobular inflammatory responses (Figure 4B). IHC analysis on F4/80 of these sections also showed an increase of infiltrating macrophages in the OGT-LKO livers (Supplemental Figure 4B). Ki-67 stains were performed, and no difference was observed between 
Table 1. Top upregulated pathways in OGT-LKO mice

\begin{tabular}{lccc}
\hline ID & Name & $\boldsymbol{P}$ value & Source \\
1270244 & ECM organization & $1.044 \times 10^{-35}$ & REACTOME \\
1269763 & Cell cycle, mitotic & $1.550 \times 10^{-20}$ & REACTOME \\
1270245 & Collagen formation & $7.601 \times 10^{-13}$ & REACTOME \\
83067 & Focal adhesion & $1.176 \times 10^{-13}$ & KEGG \\
83068 & ECM-receptor interaction & $5.046 \times 10^{-12}$ & KEGG \\
83054 & Cell cycle & $1.090 \times 10^{-8}$ & KEGG \\
& & &
\end{tabular}

WT and OGT-LKO mice, indicating that hepatocyte proliferation was not affected in the KO livers (Supplemental Figure 4C). Excessive collagen deposition in the Masson's trichrome stains and Sirius red stains revealed extensive sinusoidal fibrosis in OGT-LKO mice (Figure 4C and Supplemental Figure 4D). Pathological scoring of liver fibrosis, portal, and lobular inflammation confirmed the progression to liver fibrosis and inflammation in 10-week-old OGT-LKO mice (Figure 4D). This increase of infiltrating immune cells and ECM deposition also explain the enlargement of the livers in OGT-LKO mice. The spleen was also enlarged in OGT-LKO mice (Figure 4E), which could be partially attributed to portal hypertension caused by liver fibrosis in 10-week-old OGT-LKO mice. Ten-week-old OGT-LKO livers also showed strikingly higher hydroxyproline content as compared with WT mice, which indicated elevated ECM production in the injured livers (Figure $4 \mathrm{~F}$ ). Consistent with the pathological observations of liver fibrosis and inflammation, OGT-LKO mice showed a dramatic increase in the expression of fibrogenic (Acta2, Colla1, and Mmp2) and proinflammatory genes ( $C c l 2, I l 6$, and $T n f a$ ) (Figure 4, G and $\mathrm{H}$ ). The activation of hepatic stellate cells (HSCs) contributes significantly to the development of liver fibrosis (37). We isolated primary HSCs from WT and OGT-LKO livers and found the expression of aSMA only in OGT-LKO HSCs (Figure 4I). Primary HSCs from the KO livers also showed increased mRNA levels of Acta2, Pdgfb, and Tgfb, which indicated the activation of HSCs (Figure 4J). Collectively, OGT-LKO mice show a rapid and spontaneous progression from ballooning degeneration to liver fibrosis and inflammation within 10 weeks after birth.

OGT-deficient primary hepatocytes undergo excessive necroptosis. To identify cellular dysfunction that leads to liver injury, we isolated primary hepatocytes from 4-week-old OGT-LKO and WT mice and plated the cells on collagen-coated plates. OGT-deficient hepatocytes exhibited rounded morphology and enlarged cell size (Figure 5A and Supplemental Figure 5A). Twenty-four hours after plating, we examined the viability of isolated primary hepatocytes with Trypan blue staining and CellTiter assays. Concomitantly, viable cell number and cellular ATP content were both reduced in OGT-deficient hepatocytes, all of which represented necrotic cell death (Figure 5, B and C). In primary hepatocytes stained with propidium iodine (PI), OGT-LKO hepatocytes also showed higher PI-positive population, indicating an increase in necrosis (Supplemental Figure 5B). To further determine which cell death pathway is involved in OGT-LKO hepatocellular death, primary hepatocytes were subjected to annexin V-PI double staining and flow cytometry analysis. Compared with WT hepatocytes, a dramatic increase in the percentage of necroptotic cells was found in KO hepatocytes, whereas the apoptotic cell percentage was comparable between the 2 groups (Figure 5D). To confirm that apoptosis is not the major type of cell death in OGT-deficient hepatocytes, we examined the caspase activity in primary hepatocytes. Caspase 8 is a key mediator in the apoptosis pathway, activation of which initiates the caspase cascade and suppresses the necroptosis pathway. We found no changes in the activity of caspase 8 and its downstream targets caspase 3 and -7 , indicating a minimal contribution of apoptosis to the cell death seen in OGT-deficient hepatocytes (Supplemental Figure 5, C and D). The execution of necroptosis relies on the kinase activity of RIPK3, which could be inhibited by a selective inhibitor GSK-872. To further confirm the contribution of necroptosis in OGT-deficient hepatocytes, we treated the cells with GSK-872 and found that it significantly alleviated necroptosis in OGT-deficient hepatocytes (Figure 5, A-C). Treatment with GSK-872 also repressed the expression of phosphorylated MLKL in OGT-deficient hepatocytes (Figure 5E). In contrast, KO hepatocytes treated with the pan-caspase inhibitor z-VAD showed no difference in cell viability as compared with the nontreated group, further confirming that apoptosis is not the major cause of hepatocyte death in OGTLKO livers (Figure 5F). Mitochondrial ROS production has been shown to be associated with necroptosis in many cell types (38). We therefore tested whether mitochondrial ROS production was increased in 
Table 2. Top downregulated pathways in OGT-LKO mice

\begin{tabular}{|c|c|c|c|}
\hline ID & Name & $P$ value & Source \\
\hline 1270158 & Metabolism of amino acids and derivatives & $2.826 \times 10^{-14}$ & REACTOME \\
\hline 1270189 & Biological oxidations & $5.550 \times 10^{-12}$ & REACTOME \\
\hline 1270161 & Branched-chain amino acid catabolism & $3.023 \times 10^{-10}$ & REACTOME \\
\hline 132956 & Metabolic pathways & $4.504 \times 10^{-25}$ & KEGG \\
\hline 131226 & Peroxisome & $9.220 \times 10^{-9}$ & KEGG \\
\hline 83031 & Metabolism of xenobiotics by cytochromeP450 & $5.336 \times 10^{-7}$ & KEGG \\
\hline
\end{tabular}

KO hepatocytes and whether GSK-872 can block ROS accumulation by suppressing necroptosis. OGTLKO hepatocytes displayed more intensive MitoSox staining as compared with WT hepatocytes, which was diminished by the treatment of GSK-872 (Figure 5, G and $\mathrm{H}$ ). In contrast, antioxidants butylated hydroxyanisole (BHA) and N-Acetyl-Cysteine (NAC) failed to ameliorate necroptosis in OGT-deficient hepatocytes (Supplemental Figure 5, E and F). Taken together, these results demonstrate that deficient O-GlcNAcylation induces necroptosis in hepatocytes.

OGT negatively regulates the necroptosis pathway. RIPK3 and MLKL are key mediators of necroptosis (7). To investigate whether OGT deletion augments necroptosis by affecting RIPK3 and MLKL expression, we performed immunofluorescence staining of primary hepatocytes with antibodies against RIPK3 and MLKL. In agreement with our hypothesis, OGT-LKO hepatocytes showed increased immunofluorescence intensity of RIPK3 and MLKL staining as compared with WT hepatocytes (Figure 6A). We also found an increase in Ripk3 and Mlkl transcripts in OGT-LKO primary hepatocytes (Supplemental Figure 6A). In contrast, the mRNA expression levels of caspase 3 and caspase 8 were not changed in OGT-LKO hepatocytes (Supplemental Figure 6B). IHC staining also revealed an increased level of RIPK3 in OGT-LKO livers (Figure 6B). To confirm the activation of necroptosis in OGT-LKO mice, we examined the levels of key mediators in both the necroptosis and apoptosis pathways. Consistently, total and phosphorylated RIPK3 and MLKL proteins were increased in OGT-LKO mouse livers, whereas RIPK1, cleaved caspase 3, and cleaved caspase 8 protein levels were unchanged (Figure 6C), suggesting a specific activation of necroptosis in OGT-LKO livers. We also found similar changes in isolated primary hepatocytes (Figure 6D and Supplemental Figure 6, C-F), which indicated a cell-autonomous effect of OGT on necroptosis in hepatocytes. In multiple organs, increasing O-GlcNAcylation promotes cell survival under stress (25). To evaluate the response of hepatocyte death upon acute disruption of O-GlcNAcylation, primary hepatocytes from WT mice were transfected with scrambled siRNA or OGT siRNA to assess whether acute deletion of OGT also selectively sensitized hepatocytes to necroptosis but not apoptosis. Apoptosis was induced by treating the cells with TNFA and cycloheximide (CHX) for 24 hours, and OGT-ablated hepatocytes showed a similar response to the treatment as compared with the control group (Supplemental Figure 6G). The pan-caspase inhibitor z-VAD was added with TNFA and CHX for 24 hours to induce hepatocyte necroptosis. Hepatocyte viability was significantly decreased in siOGT-transfected cells but not in the control group when necroptosis was induced (Figure 6E). Treatment of GSK-872 suppressed necroptosis observed in OGT-deficient hepatocytes (Figure 6E). Increased RIPK3 and MLKL protein levels were only seen in OGT siRNA-transfected hepatocytes, indicating that elevated RIPK3 and MLKL expression underlies the vulnerability to necroptotic treatments upon acute OGT deletion (Figure 6F). To examine whether inhibition of necroptosis also alleviates liver damage in vivo, we injected a selective necroptosis inhibitor necrostatin 1 stable (Nec-1s) into 3-week-old WT and OGT-LKO mice for 1 week. We observed significant improvement of liver injury in OGT-LKO mice with Nec-1s treatment, as demonstrated by reduced liver/body weight ratio, decreased ALT levels, and decreased hepatocyte ballooning (Figure 6, G-I). Taken together, these data indicate that OGT is a negative regulator of the necroptosis pathway and associated liver injury.

OGT glycosylates RIPK3 and decreases RIPK3 protein stability. We next sought to dissect the molecular mechanisms underlying OGT regulation of necroptosis. The balance of different PTMs, including O-GlcNAcylation, phosphorylation, and ubiquitination, precisely controls the expression and activity of their substrates. Previous studies have shown that RIPK3 is modified by both phosphorylation and ubiquitination $(39,40)$. We found abundant RIPK3 O-GlcNAcylation in WT mouse livers but to a much lower level in OGT-LKO mouse livers (Figure 7A). To determine whether OGT interacts with RIPK3, HEK293T 
A

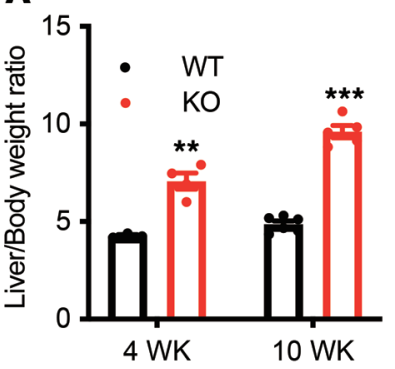

B

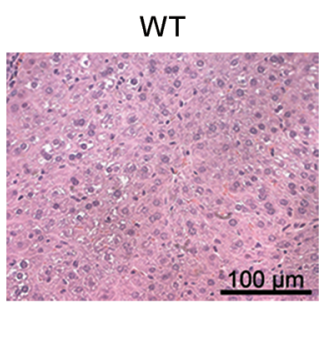

C

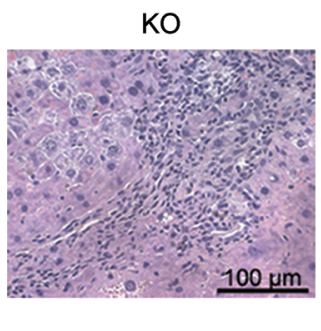

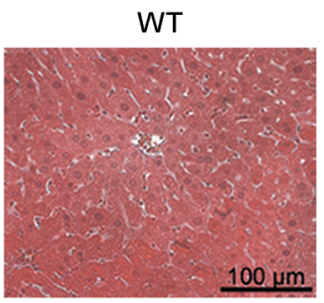

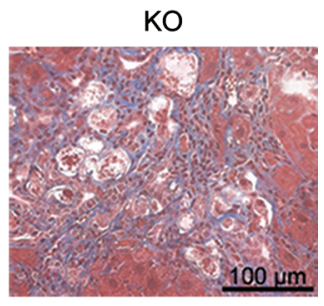

D

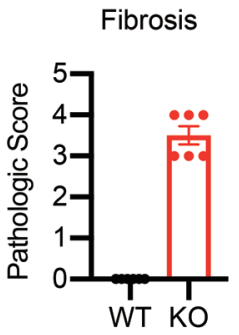

Portal Inflammation

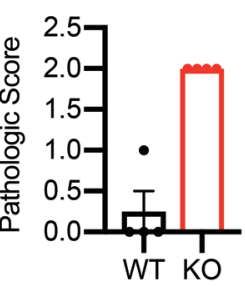

Lobular Inflammation

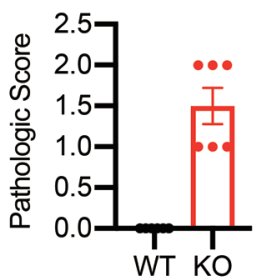

E

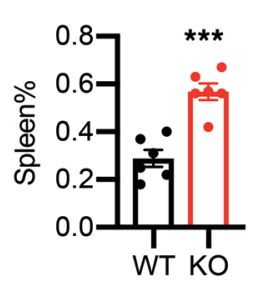

\section{$\mathbf{F}$}

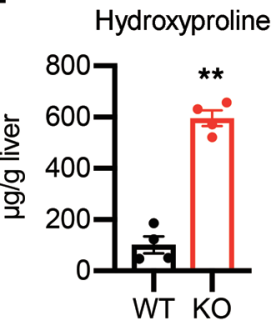

G

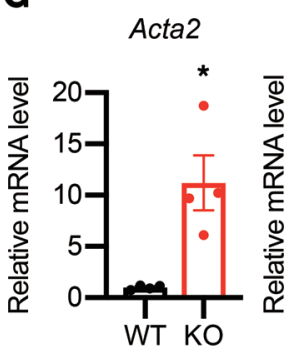

Col1a1

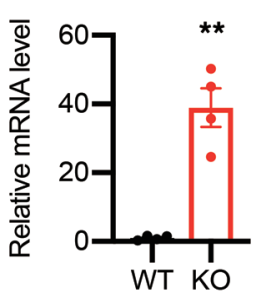

Mmp2

H

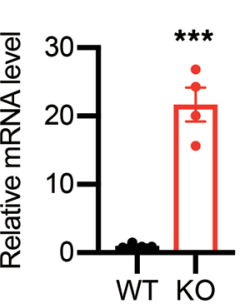

Cc/2

116

Tnfa
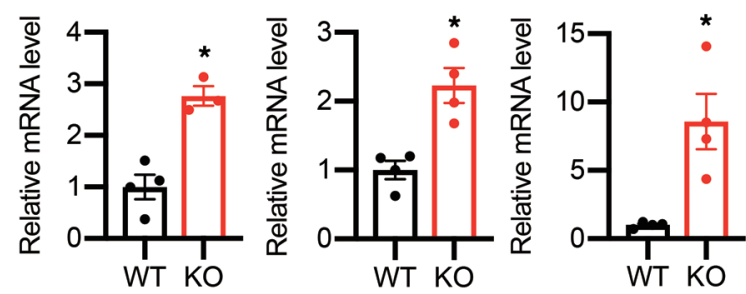

I

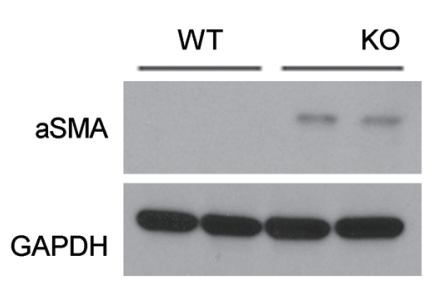

J

Acta2

Pdgfb

Tgfb
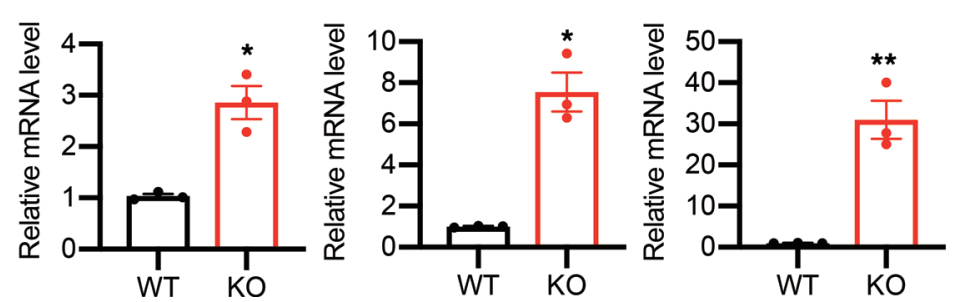

Figure 4. OGT-LKO mice develop liver fibrosis and inflammation at 10 weeks of age. (A) Percentage of liver weight to body weight in 4- and 10-week-old mice. (B) H\&E stains showing immune cell infiltration in OGT-LKO livers. (C) Masson's trichrome stains showing extracellular matrix deposition in OGT-LKO livers. (D) Pathologic scores for liver fibrosis and portal and lobular inflammation. (E) Percentage of spleen weight to body weight. (F) Hydroxyproline content in the liver. (G) Expression levels of fibrogenic genes. (H) Expression levels of proinflammatory genes. (I) Western blotting of proteins from primary hepatic stellate cells (HSCs) showing the expression levels of $\alpha$ SMA. GAPDH was used as loading control. (J) Expression levels of Acta2, Pdgfb, and Tgfb in primary HSCs isolated from WT and OGT-LKO mice. $n=4$, both sexes. Data are shown as mean \pm SEM. ${ }^{*} P<0.05 ;{ }^{* *} P<0.01$; ${ }^{* *} P<0.001$ by unpaired Student's $t$ test. OGT-LKO, liver-specific OGT KO; $\alpha \mathrm{SMA}, \alpha$ smooth muscle actin; Acta2, smooth muscle $\alpha 2$ actin; Pdgfb, platelet derived growth factor subunit B; Tgfb, transforming growth factor b.

cells were cotransfected with Myc-OGT and FLAG-RIPK3. Immunoprecipitation followed by Western blotting revealed the binding of OGT and RIPK3 (Figure 7B). To understand how OGT affects RIPK3 protein expression, increasing doses of the OGT-expressing plasmid were transfected into HEK293T cells. The result showed a dose-dependent decrease in RIPK3 protein levels with increasing OGT expression (Figure 7C). To test whether glycosylation affects the protein stability of RIPK3, a specific OGA inhibitor, Thiamet-G (TMG), was used to increase intracellular O-GlcNAc levels. Concomitantly, TMG effectively shortened the half-life of RIPK3 in H1299 cells as compared with the vehicle control (Figure 7D). Collectively, these results indicate that O-GlcNAcylation of RIPK3 decreases its protein stability and expression. 
A

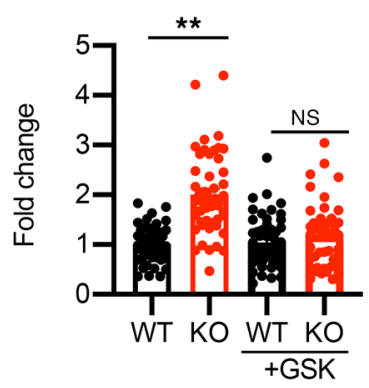

B

Cell viability

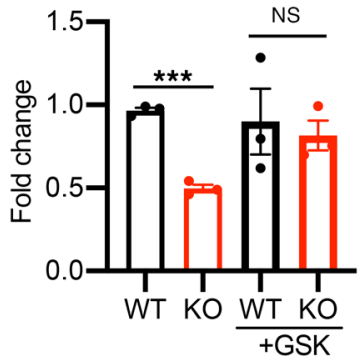

C ATP content

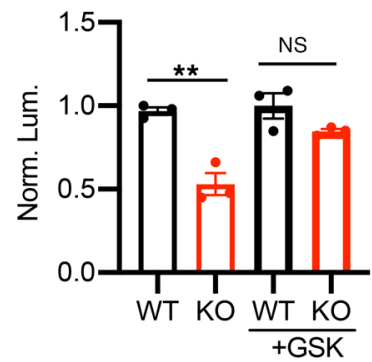

D

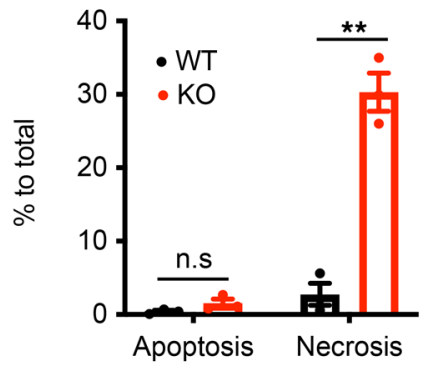

E

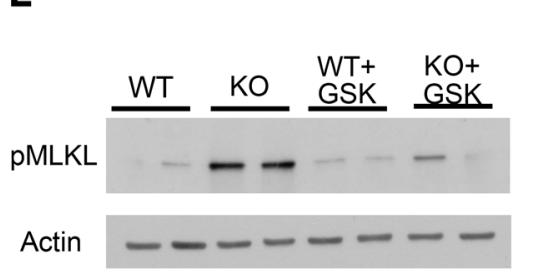

F

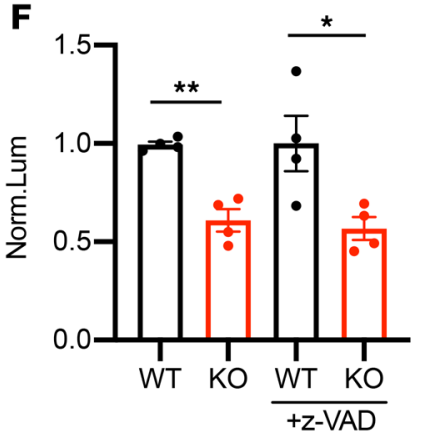

H

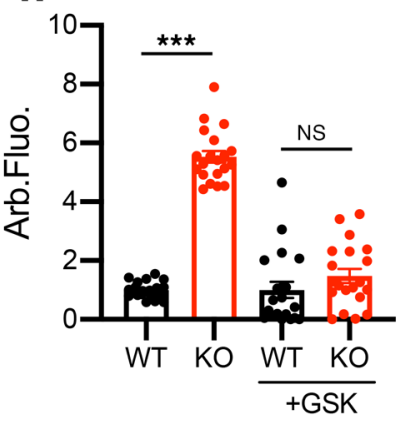

G
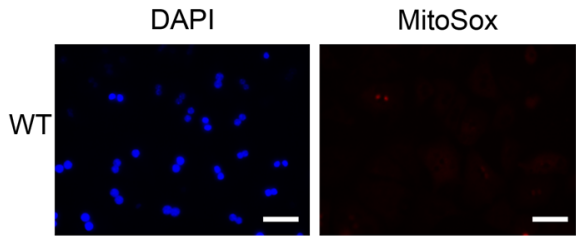

Overlap
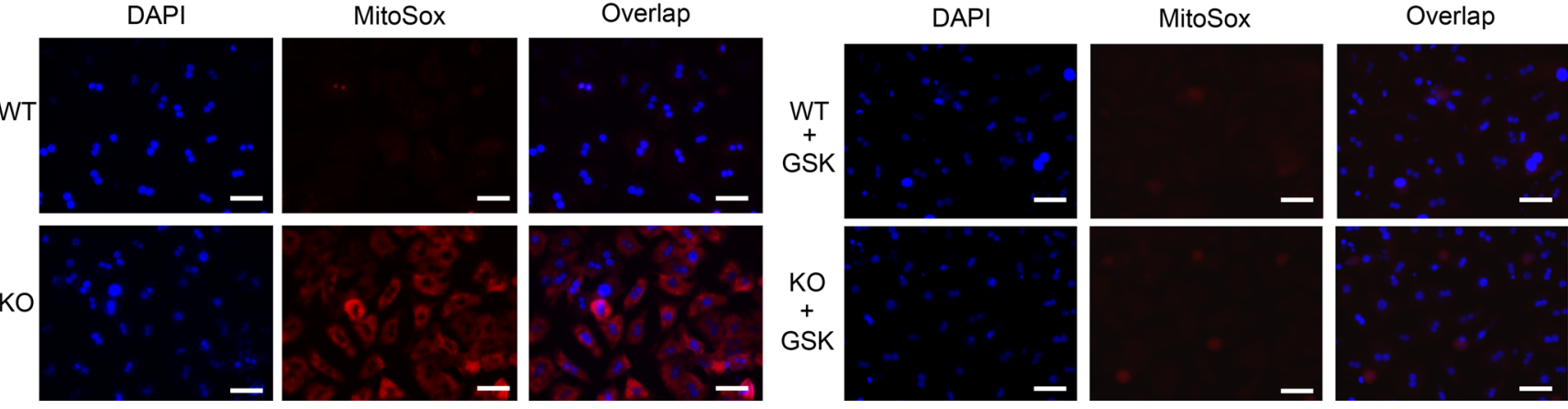

Figure 5. Loss of OGT in hepatocytes leads to excessive necroptosis. (A) Primary hepatocyte cell size quantified under light microscopy ( $n=50)$. (B) Primary hepatocyte viability determined by Trypan blue staining $(n=3)$. (C) ATP content determined by the CellTiter-Glo luminescent cell viability assay kit ( $n$ = 3). Data were normalized to cell number. (D) Flow cytometry analysis of annexin V-propidium iodine (PI) double-stained primary hepatocytes. Data were normalized to total cell number $(n=3)$. (E) Western blotting of phosphorylated MLKL in primary hepatocytes. (F) Primary hepatocyte cell viability. z-VAD is a pan-caspase inhibitor $(n=4)$. ( $(\mathbf{G}$ and $\mathbf{H})$ Images and quantification of MitoSox stains of primary hepatocytes. Fluorescence intensity was quantified with ImageJ $(n=20)$. Scale bar: $50 \mu \mathrm{m}$. Data are shown as mean $\pm \mathrm{SEM} .{ }^{*} P<0.05 ;{ }^{* *} P<0.01 ;{ }^{* *} P<0.001$ by unpaired Student's $t$ test. OCT, 0 -GIcNAc transferase; GSK, GSK-872, RIPK3 kinase inhibitor; RIPK3, receptor-interacting protein kinase 3; MLKL, mixed lineage kinase domain-like.

\section{Discussion}

Liver fibrosis and cirrhosis is the common path to hepatocellular carcinoma (HCC), which is among the deadliest cancers around the world. Hepatocellular death exists in almost all acute and chronic liver pathologies, acting through different cell death pathways to initiate either phagocytosis, compensatory proliferation, or inflammation and fibrosis. Here, we report that OGT-deficient hepatocytes undergo massive necroptosis, which contributes to the fast-developing liver injury manifested by hepatocyte ballooning, ALT elevation, inflammation, and liver fibrosis (Figure 7E). Moreover, apoptosis is not evident in our model, indicating that necroptosis itself could contribute significantly to the pathogenesis of liver injury. Compared with other forms of cell death, necroptosis represents a more inflamed mode, raising a higher possibility of tissue inflammation that can initiate subsequent fibrogenic events. This phenomenon is well exemplified in our model, as we observed the progression of liver injury in OGTLKO mice from ballooning degeneration to fibrosis, which mirrors the progressive nature of chronic 
A
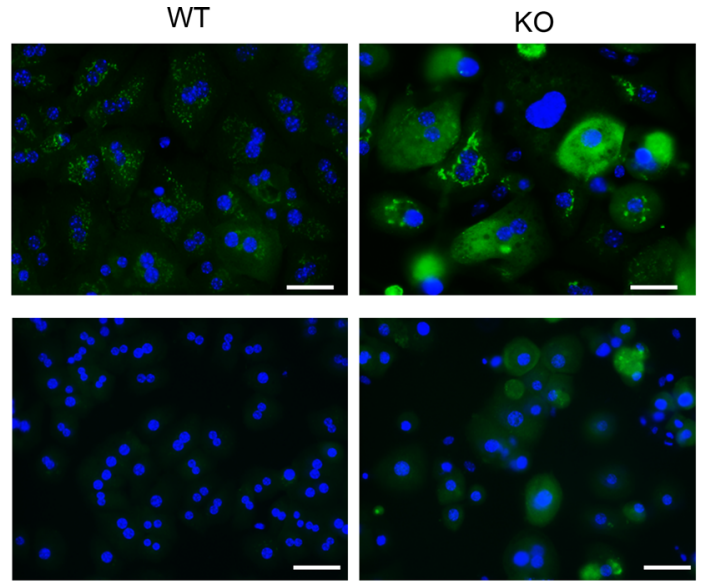

Liver extracts

C

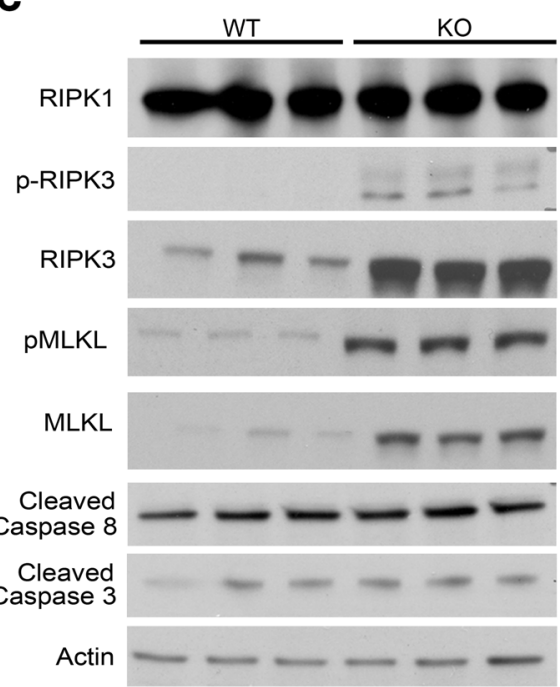

D

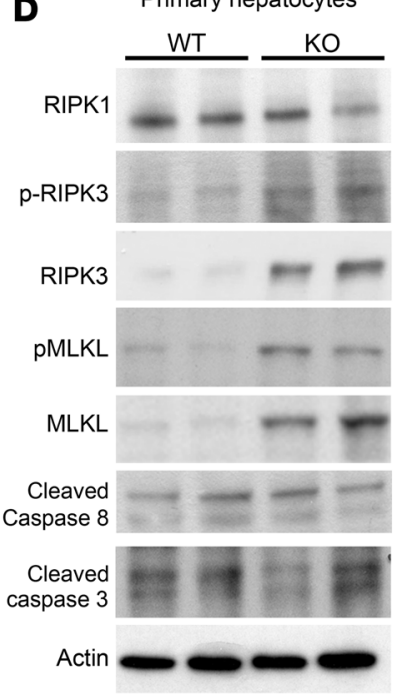

B
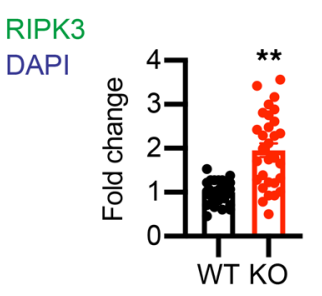

MLKL
DAPI

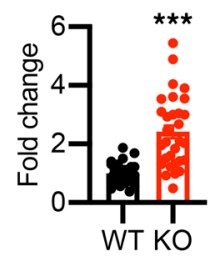

$\mathrm{KO}$
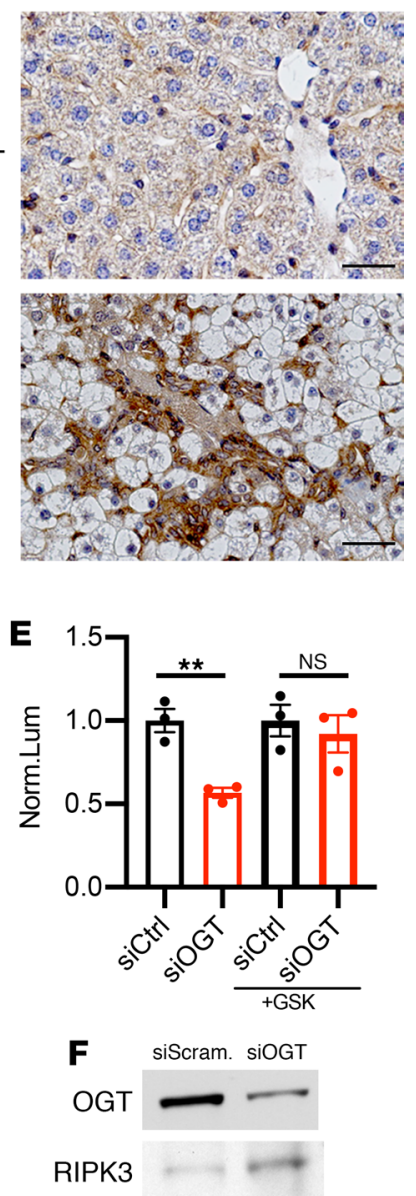

MLKL

Actin
G

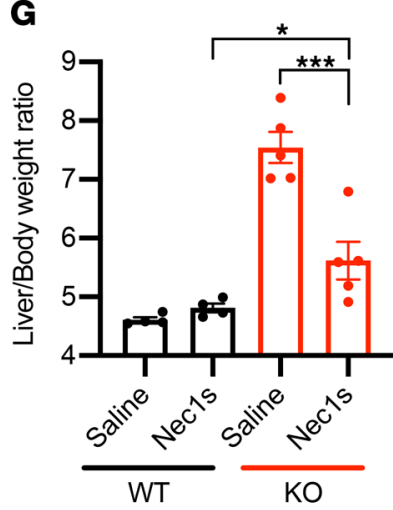

H

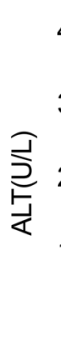

$\left.\begin{array}{l}400 \\ 300 \\ 200- \\ 100 \\ 0\end{array}\right]$
I

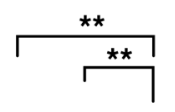

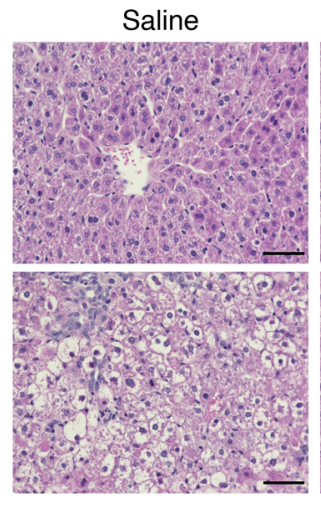

Nec-1s

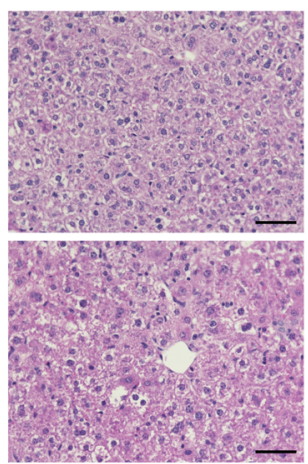

Figure 6. OGT deletion induces the expression of RIPK3 and MLKL in primary hepatocytes. (A) Immunofluorescence images of primary hepatocytes stained with antibodies against RIPK3 and MLKL (green). The nucleus was stained with DAPI (blue). Fluorescence intensity was quantified with Image) ( $n$ = 30). (B) IHC stains of RIPK3 in liver sections from 4-week-old WT and OCT-LKO mice. (C and D) Western blotting of proteins from liver extracts (C) and primary hepatocytes (D) showing the expression levels of RIPK1, phosphorylated and total RIPK3, phosphorylated and total MLKL, and cleaved caspase 8 and caspase 3. Actin was used as loading control. (E) WT primary hepatocytes transfected with scrambled siRNA or OCT siRNA were treated with TNFA, cycloheximide (CHX), and z-VAD for 24 hours to induce necroptosis. Cell viability was determined with CellTiter-Glo luminescent cell viability assay kit ( $n=$ 3). Data were normalized to the DMSO-treated group. (F) Western blotting of RIPK3 and MLKL in primary hepatocytes transfected with scrambled siRNA or OGT siRNA and treated with TNFA, CHX, and z-VAD. Actin was used as loading control. (G) Liver weight to body weight ratio of saline or Nec-1s-treated mice. (H) ALT levels of saline or Nec-1s-treated mice. (I) H\&E stains of saline or Nec-1s-treated mouse livers. All primary hepatocyte experiments were repeated at least 3 times with different cohorts of mice. Scale bar: $50 \mu \mathrm{m}$. Data are shown as mean $\pm \mathrm{SEM}$. ${ }^{*} P<0.05 ;{ }^{* *} P<0.01 ;{ }^{* * *} P<0.001$ by unpaired Student's $t$ test. OGT, O-GICNAc transferase; RIPK3, receptor-interacting protein kinase 3; MLKL, mixed lineage kinase domain-like; OCT-LKO, liver-specific OCT KO; RIPK1, receptor-interacting protein kinase 1; CHX, cycloheximide; Nec-1s, necrostatin 1 stable; ALT, alanine aminotransferase. 


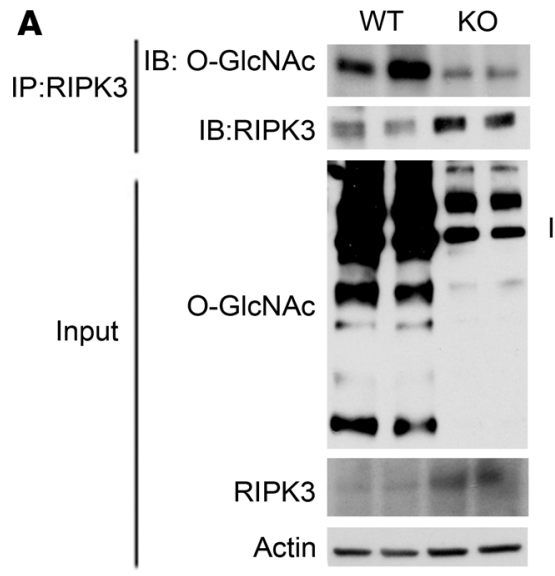

D

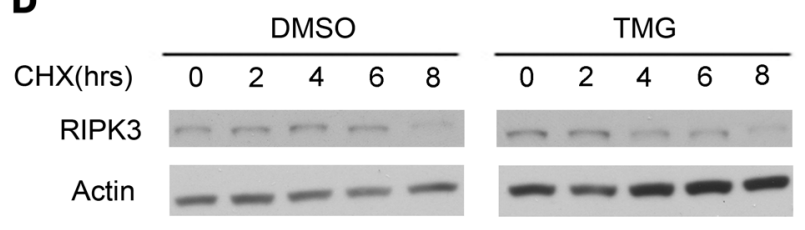

$\begin{array}{cc}\text { B } & \begin{array}{c}\text { Vector } \\ \text { Myc-OGT } \\ \text { FLAG-RIPK3 }\end{array} \\ \text { IP: FLAG } & \text { IB: Myc } \\ \text { IB: FLAG }\end{array}$

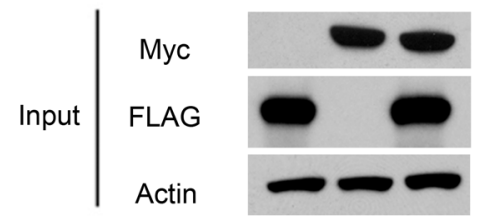

C

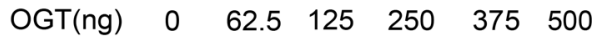

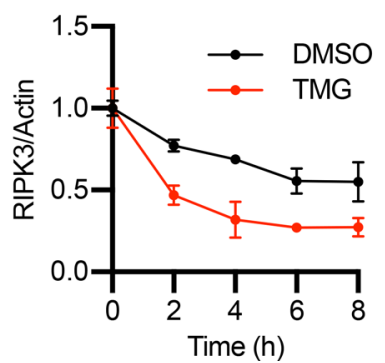

E Insults

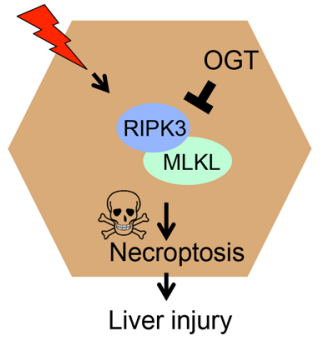

Figure 7. OGT glycosylates RIPK3 and regulates RIPK3 protein stability. (A) O-GIcNAcylation of RIPK3 in WT and OGT-LKO livers are shown. (B) HEK293T cells were cotransfected with Myc-OGT and FLAG-RIPK3, and their interaction was determined. Empty vector plasmid was transfected to keep equal transfection amount in each group. (C) HEK293T cells were cotransfected with RIPK3 and various doses of OGT as indicated. Levels of RIPK3 and OGT were determined by Western blots. (D) RIPK3-transfected H1299 cells were pretreated with DMSO or TMC and then with CHX for indicated time points. Stability of RIPK3 was determined with Western blots and quantified with ImageJ. (E) Model for hepatocyte OGT function in protecting against liver injury by suppressing RIPK3/MLKL-mediated necroptosis. All experiments were repeated at least twice. OCT, 0-CIcNAc transferase; OGT-LKO, liver-specific OGT KO; RIPK3, receptor-interacting protein kinase 3; TMG, thiamet-G; CHX, cycloheximide.

liver diseases in patients. However, we noticed that pharmacological inhibition of necroptosis by Nec-1s only partially ameliorated liver injury in OGT-LKO mice, suggesting that other pathways downstream of OGT deficiency also contribute to fibrogenic liver pathologies.

It has very recently been reported that, besides apoptosis, necroptosis is involved in the etiology of $\mathrm{ASH}, \mathrm{NASH}$, and viral infections (3). We observed a robust activation of necroptosis in patients with alcoholic liver cirrhosis and in mice fed ethanol diet, confirming a critical role for this cell death pathway in chronic liver diseases. In contrast, the contribution of necroptosis to acute liver failure is under debate. Emerging evidence suggests that necroptosis may not be involved in drug-induced acute liver injury (4). It seems that prolonged disruption of the balance between hepatocyte survival and death is a critical determinant for chronic liver disease but not in acute liver failure. A recent paper showed that systemic administration of the OGA inhibitor exacerbates acetaminophen-induced liver injury, and hepatic depletion of OGT with AAV protects the mice from such injury (41). The discrepancies between this study and our current study may result from the different methods used to manipulate O-GlcNAc signaling in the liver. As O-GlcNAcylation is a highly dynamic PTM, acute or chronic changes in O-GlcNAc signaling may lead to different outcomes by modulating different substrates (22). It is also noteworthy that, in our mouse model of ethanol diet-induced liver injury, hepatocyte injury and inflammation were evident, while no obvious fibrosis was developed before the mice were sacrificed (34). The levels of liver O-GlcNAcylation were already decreased significantly in these mice. These results suggest that O-GlcNAcylation responds to liver stress at an early stage of chronic liver disease. It will be important to determine whether O-GlcNAc could serve as a biomarker for liver disease in future studies.

In our study, we showed that the expression of RIPK3 is induced in OGT-LKO livers and primary hepatocytes, whereas the expression and activities of caspases are unchanged as compared with WT primary hepatocytes. Previous studies have proposed that an intricate balance and mutual regulation between apoptosis and necroptosis help maintain tissue homeostasis, and suppressing either of them 
will cause the upregulation in the other $(42,43)$. The molecular basis for this is that the necroptosis and apoptosis pathways utilize overlapping signaling components, such as the protein complex assembled upon death receptor binding. However, the 2 pathways diverge downstream of RIPK1, where either caspase 8 or RIPK3 is activated. In the liver, studies have shown that the reciprocal inhibition of caspase 8 and RIPK3 is critical for the development of NASH and hepatocarcinogenesis $(6,44)$. RIPK 3 inhibits the cleavage of caspase 8 and subsequent activation of JNK to stall cell proliferation and, thus, limit HCC development (44). Interestingly, a previous study by Xu et al. demonstrated that overexpression of OGT in the liver promotes HCC through activation of JNK (45). These results, along with ours, indicate that OGT is prosurvival in the hepatocytes, ablation of which not only results in uncontrolled necroptosis, but may also compromise cell proliferation. Further investigations into the role of OGT in other chronic liver injury models would elucidate how OGT is integrated in this signaling network.

It has long been known that PTMs such as glycosylation, phosphorylation, and ubiquitination add a critical layer of regulation on protein functions. Here, we have demonstrated that O-GlcNAcylation reduces RIPK3 protein stability and suppresses its expression. Additionally, Ripk3 and Mlkl mRNA levels are also increased in OGT-deficient hepatocytes. These results indicate that O-GlcNAcylation affects the necroptosis pathway at both transcriptional and posttranslational levels. Dissecting the molecular mechanisms by which OGT regulates RIPK3 gene transcription and protein turnover in hepatocytes will be an important subject for future investigation, which will shed light on how healthy livers keep necroptosis in check.

Liver fibrosis is a critical stage in chronic liver disease, and there are no effective antifibrotic therapies clinically available. One of the challenges in developing antifibrotic drugs is the lack of effective animal models. A majority of current mouse models are established with a special diet or toxic reagents. It takes at least several months to develop liver fibrosis, and the phenotypes are usually irreversible. Therefore, many features in human liver fibrosis cannot be faithfully recapitulated, and large-scale, long-term studies are often impossible in these models. Available genetic models of liver fibrosis are mostly whole body-KO mice, such as $M d r 2^{--}$(46), Il-2R $R^{-/}$(47), or dominant-negative Tgf $\beta$ receptor $\Pi$ (Tgf- $\beta$ RII) mice (48), which have confounding effects ascribed to other tissues. Our tissue-specific OGT-LKO mice spontaneously develop liver fibrosis in less than 10 weeks after birth. Therefore, the OGT-LKO model can potentially be employed as a novel, effective mouse model of liver fibrosis with broad translational implications for antifibrotic drug screening and evaluation.

\section{Methods}

Animal studies. Ogftoxed mice on C57BL/6J background were provided by Steven Jones at the University of Louisville (Louisville, Kentucky, USA) (49). Alb-Cre mice were provided by Anton Bennett at Yale University. All mice were kept on a 12-hour light, 12-hour dark cycle. All mice had free access to food and water. Both sexes were included in the experiments, and the age of mice used is specified in the text. Mice were fasted for 6 hours before sacrifice, and tissues were collected for protein and for RNA extraction and histology analysis. Ethanol-induced liver injury was induced as described before (34). Briefly, mice were fed with a Lieber-DeCarli liquid diet (F1258SP, BioServ) or a control diet (F1259SP, BioServ) that has a matched calorie with ethanol diet for 6 weeks. Nec-1s was dissolved in DMSO (final concentration, $8 \%$, v/v), PEG (final concentration, 8\%, v/v), and saline stock solution, which was further diluted with warm saline to final concentration before injection. Vehicle control or Nec-1s was administered at the dose of $15 \mathrm{mg} / \mathrm{kg} /$ day through i.p. injection repetitively for a week.

Metabolic assays. Body weights were measured each week. Metabolic cage study was carried out with the Promethion multiplexed metabolic measurement system. Mice were single-housed for 5 days and acclimated in metabolic chambers for 3 days before the measurement of gas exchange, food intake, and ambulatory activity.

Histology. Mouse livers were dissected and fixed in 4\% paraformaldehyde for 48 hours. H\&E stains, Masson's trichrome stains, and Sirius red stains were performed by the Histology Core in the Department of Comparative Medicine at Yale University. IHC was performed with the Vectastain Elite ABC HRP kit (Vector Laboratories). Paraffin-embedded sections were deparaffined, and antigen retrieval was carried out in a preheated steamer. The slides were then blocked for background peroxidase activity with hydrogen peroxide solution and for unspecific binding with blocking buffer provided in the kit. The slides were incubated with 1:50 primary antibody overnight at $4^{\circ} \mathrm{C}$ and secondary antibody for 30 minutes at room temperature. The slides were counterstained with Mayer's hematoxylin solution, dehydrated, and mounted. 
ELISA. Liver tissues were weighed and homogenized in assay diluent provided by the TNFA or IL-6 ELISA kit (BD Biosciences). Plasma samples were collected with heparin as anticoagulant, centrifuged at 1,000 $g$ at $4^{\circ} \mathrm{C}$, and stored at $-80^{\circ} \mathrm{C}$ until measurement. Assays were performed according to the manufacturer's protocol.

RNA-seq. mRNA was extracted from snap-frozen liver tissues with QIAGEN RNeasy kit. Library preparation and sequencing were carried out at Novogene on the Illumina NovaSeq 6000 system with total raw reads at 20 million per sample, on average. RNA-seq data analysis was performed in collaboration with Dongjun Chung's group at the Medical University of South Carolina. These data are available at Gene Expression Omnibus with the accession number GSE134993.

Flow cytometry. Freshly isolated primary hepatocytes were washed twice with BioLegend's cell staining buffer and then resuspended in annexin $\mathrm{V}$ binding buffer at the density of $5 \times 10^{6}$ cells $/ \mathrm{mL}$. A $100-\mu \mathrm{L}$ cell suspension was transferred to the test tube and stained with $5 \mu \mathrm{L}$ Pacific Blue annexin V and PI solution (BioLegend) for 15 minutes each in the dark. A $400-\mu \mathrm{L}$ annexin $\mathrm{V}$ binding buffer was then added to the test tube, and the samples were analyzed with BD LSR II flow cytometer.

Fluorescent imaging and live cell imaging. Primary hepatocytes were plated on 8-well chamber slides for immunofluorescence imaging and on collagen-coated 6-well plates (BD Biosciences) for PI stains and MitoSox stains. For immunofluorescence, primary hepatocytes were fixed with $4 \%$ paraformaldehyde for 20 minutes and permeabilized in 0.5\% Triton X-100 (American Bioanalytical) for 10 minutes at room temperature. Cells were blocked with $2 \%$ BSA in PBST $(0.5 \%$ Tween 20 in PBS) and then incubated with primary antibodies at $4^{\circ} \mathrm{C}$ overnight in a humidified chamber. The cells were incubated with Alexa Fluor 488-conjugated secondary antibodies (Invitrogen) for 1 hour at room temperature in the dark, followed by staining with DAPI. The slides were mounted with Vectashield antifade mounting medium (Vector Laboratories). For PI staining, primary hepatocytes were washed with PBS and incubated with PI (BioLegend) in the dark at room temperature for 5 minutes. MitoSox Red (Thermo Fisher Scientific) staining was performed according to the manufacturer's protocol. Quantification of fluorescence was carried out with ImageJ software (NIH).

Cell viability assays. The CellTiter-Glo luminescent cell viability assay kit (Promega) was used to determine the viability of primary hepatocytes. The cells were plated in black-wall, clear-bottom 96-well plate at the density of $3 \times 10^{4}$ cells/well. Four hours after plating, the cells were treated with various drugs as indicated in the text for 24 hours. The plates were brought to room temperature for 30 -minute equilibration, and $100 \mu \mathrm{L}$ assay buffer was added to each well. The plates were incubated for 10 minutes, and luminescence was determined with the Tecan Infinite M200 plate reader.

Cell culture. HEK293T and H1299 cells were cultured in DMEM with 10\% FBS (Thermo Fisher Scientific). Primary hepatocytes and primary HSCs from WT and OGT-LKO mice were isolated by Yale Liver Center Core Facility. Primary hepatocytes were cultured in Williams' Medium E supplemented with 10\% FBS, 10 mM HEPES buffer, $2 \mathrm{mM}$ L-glutamine, $8 \mathrm{mg} / \mathrm{L}$ gentamicin, SPA, $1 \mu \mathrm{M}$ dexamethasone, $4 \mu \mathrm{g} / \mathrm{mL}$ insulin, and $1 \mathrm{mM}$ glucose. All primary hepatocytes were plated on Collagen I-coated plates (BD Biosciences). Transfection of plasmids or siRNAs was performed with FuGene HD (Promega) or Lipofectamine RNAimax (Invitrogen), respectively. DMEM, FBS, William's medium E, HEPES buffer, glutamine stock solution, SPA, gentamicin, amphotericin were from Thermo Fisher Scientific. Dexamethasone, insulin, and glucose were purchased from Sigma-Aldrich. H1299 cells were provided by Qin Yan at Yale University.

Plasmids and siRNAs. Myc-OGT was provided by Xiaochun Yu at the University of Michigan. The mRIPK3-GFP plasmid was purchased from Addgene. FLAG tag at the C-terminal of human RIPK3 was cloned into the pEGFP-N1 vector. Scrambled siRNA (5'-GAGGCAUGUCCGUUGAUUCGU-3') and OGT siRNA (5'-GAGGCAGUUCGCUUGUAUCGU -3') were synthesized by Dharmacon.

Real-time PCR. Total RNA was extracted from cells or tissues with TRIzol reagent (Invitrogen). Complementary DNA was synthesized from total RNA with Superscript III enzyme (Bio-Rad) and amplified with SYBR Green Supermix (Bio-Rad) using a LightCycler480 Real-Time PCR system (Roche). All data were normalized to the expression of 36B4 mRNA levels. Primer sequences are listed in Supplemental Table 2.

Western blotting and immunoprecipitation. Liver tissues or cells were lysed in buffer containing $1 \%$ Nonidet P-40, $150 \mathrm{mM} \mathrm{NaCl}, 0.1 \mathrm{mM}$ EDTA, $50 \mathrm{mM}$ Tris- $\mathrm{HCl}$, proteinase inhibitors, and protein phosphatase inhibitors (Sigma-Aldrich). In immunoprecipitation assays, cell lysates were mixed with various antibodies and precipitated with protein A/G agarose beads (Santa Cruz Biotechnology Inc.) or FLAG M2 monoclonal antibody affinity gel (Sigma-Aldrich) overnight at $4^{\circ} \mathrm{C}$. The lysates were then washed and boiled in SDS loading buffer. Equal amounts of protein lysates were resolved on SDS-PAGE gels and transferred to PVDF membrane. The membranes were blocked in 5\% BSA and incubated with various primary antibodies 
overnight at $4^{\circ} \mathrm{C}$. After 3 washes, the membranes were incubated with peroxidase-conjugated secondary antibodies for 1 hour and visualized with ECL chemiluminescent substrate. Quantification of western blot results was carried out with ImageJ software.

Antibodies and reagents. Antibodies against OGT (ab96718), O-GlcNAc (RL2, ab2739), mouse pMLKL S345 (ab196436), Ki-67 (ab 16667), and aSMA (ab7817) (all from Abcam); cleaved caspase 3 (9664), cleaved caspase 8 (8592), RIPK1 (3493), human pMLKL T357/S358 (14516), FLAG (2368), tubulin (2128), and GAPDH (2118) (all from Cell Signaling Technology); Myc (sc-40) and actin (sc-8432) (both from Santa Cruz Biotechnology Inc.); OGA (Novus Biologicals, NBP1-96679); and F4/80 (14-480182 , eBioscience) were purchased from the indicated sources. Antibodies against RIPK3, p-RIPK3, and MLKL were provided by Vishva Dixit from Genentech (lot NV0019934). Alexa Fluor 488-conjugated secondary antibodies were obtained from Thermo Fisher Scientific. DAPI, Trypan blue solution, and CHX were purchased from Sigma-Aldrich. Recombinant mouse TNFA was from R\&D systems. TMG and z-VAD OMe-FMK were from Cayman Chemical. Nec-1s was from BioVision. Hydroxyproline assay kit (Sigma-Aldrich) and ALT activity assay kit (Cayman Chemical) were purchased from indicated sources, and the assays were performed according to the manufacturer's protocol.

Statistics. Results are presented as mean \pm SEM. The comparisons were carried out using 2-tailed unpaired Student's $t$ test or 1-way ANOVA followed by Tukey's adjusted multiple comparisons. Data were plotted with GraphPad Prism. A $P$ value less than 0.05 was considered significant. Statistical tests used are stated in the figure legends.

Study approval. All animal procedures have been approved by the IACUC of Yale University. The human liver samples were provided by LW at the University of Connecticut. The samples were obtained from Liver Tissue Cell Distribution System (Minneapolis, Minnesota, USA), as described before (50).

\section{Author contributions}

The experiments were designed by BZ, MDL, and XY. Animal studies were performed by BZ, MDL, RY, YY, and RL. RIPK3 protein stability assays were performed by BZ and YL. RNA-seq data were processed and analyzed by JHN and DC. Histology analyses were performed by KAMR and MER. The rest of the studies were performed by BZ. Human liver samples were provided by LW. Ethanol diet mouse liver samples were provided by YI. Data analysis and interpretation were performed by BZ, MDL, YL, and XY. The manuscript was written by BZ and XY, with editing and discussion with BEE, AMB, JY, and MHN.

\section{Acknowledgments}

We thank Xiaochun Yu from the University of Michigan for providing the Myc-OGT plasmid and Vishva Dixit at Genentech for providing the antibodies. We thank Qin Yan for providing H1299 cells. We thank Kathy Harry, Masatake Tanaka, Jittima Weerachayaphorn, and Mateus T. Guerra from Yale University Liver Center for their help with experimental procedures. This work was supported by NIH (R01DK089098, R01DK102648), Yale Liver Center (P30 DK34989), American Cancer Society (RSG-14-244-01-TBE), and American Diabetes Association (1-19-IBS-119) grants to XY; NIH award P01-DK57751 to AMB, BEE, MER, MHN, and XY; and the China Scholarship Council-Yale World Scholars fellowship to BZ.

Address correspondence to: Xiaoyong Yang, 333 Cedar Street, PO Box 208016, New Haven, Connecticut 06520-8016, USA. Phone: 203.737.1446; Email: xiaoyong.yang@yale.edu.

1. Luedde T, Kaplowitz N, Schwabe RF. Cell death and cell death responses in liver disease: mechanisms and clinical relevance. Gastroenterology. 2014;147(4):765-783.e4.

2. Linkermann A, Stockwell BR, Krautwald S, Anders HJ. Regulated cell death and inflammation: an auto-amplification loop causes organ failure. Nat Rev Immunol. 2014;14(11):759-767.

3. Nagy LE, Ding WX, Cresci G, Saikia P, Shah VH. Linking Pathogenic Mechanisms of Alcoholic Liver Disease With Clinical Phenotypes. Gastroenterology. 2016;150(8):1756-1768.

4. Schwabe RF, Luedde T. Apoptosis and necroptosis in the liver: a matter of life and death. Nat Rev Gastroenterol Hepatol. 2018;15(12):738-752.

5. Afonso MB, et al. Necroptosis is a key pathogenic event in human and experimental murine models of non-alcoholic steatohepatitis. Clin Sci. 2015;129(8):721-739.

6. Gautheron J, et al. A positive feedback loop between RIP3 and JNK controls non-alcoholic steatohepatitis. EMBO Mol Med 2014;6(8):1062-1074.

7. Galluzzi L, et al. Molecular mechanisms of cell death: recommendations of the Nomenclature Committee on Cell Death 2018. 
Cell Death Differ. 2018;25(3):486-541.

8. Linkermann A, Green DR. Necroptosis. N Engl J Med. 2014;370(5):455-465

9. Kaczmarek A, Vandenabeele P, Krysko DV. Necroptosis: the release of damage-associated molecular patterns and its physiological relevance. Immunity. 2013;38(2):209-223.

10. Zhu K, et al. Necroptosis promotes cell-autonomous activation of proinflammatory cytokine gene expression. Cell Death Dis. 2018;9(5):500.

11. Dara L, Liu ZX, Kaplowitz N. Questions and controversies: the role of necroptosis in liver disease. Cell Death Discov. 2016;2:16089.

12. Günther C, et al. The pseudokinase MLKL mediates programmed hepatocellular necrosis independently of RIPK3 during hepatitis. J Clin Invest. 2016;126(11):4346-4360.

13. Afonso MB, et al. Activation of necroptosis in human and experimental cholestasis. Cell Death Dis. 2016;7(9):e2390

14. Seo MJ, Hong JM, Kim SJ, Lee SM. Genipin protects d-galactosamine and lipopolysaccharide-induced hepatic injury through suppression of the necroptosis-mediated inflammasome signaling. Eur J Pharmacol. 2017;812:128-137.

15. Dara L, et al. Receptor interacting protein kinase 1 mediates murine acetaminophen toxicity independent of the necrosome and not through necroptosis. Hepatology. 2015;62(6):1847-1857.

16. Roychowdhury S, et al. Receptor interacting protein 3 protects mice from high-fat diet-induced liver injury. Hepatology. 2016;64(5):1518-1533

17. Torres CR, Hart GW. Topography and polypeptide distribution of terminal N-acetylglucosamine residues on the surfaces of intact lymphocytes. Evidence for O-linked GlcNAc. J Biol Chem. 1984;259(5):3308-3317.

18. Hanover JA, Krause MW, Love DC. Bittersweet memories: linking metabolism to epigenetics through O-GlcNAcylation. Nat Rev Mol Cell Biol. 2012;13(5):312-321.

19. Yang X, Qian K. Protein O-GlcNAcylation: emerging mechanisms and functions. Nat Rev Mol Cell Biol. 2017;18(7):452-465.

20. Hart GW, Housley MP, Slawson C. Cycling of O-linked beta-N-acetylglucosamine on nucleocytoplasmic proteins. Nature. 2007;446(7139):1017-1022.

21. Jóźwiak P, Forma E, Bryś M, Krześlak A. O-GlcNAcylation and Metabolic Reprograming in Cancer. Front Endocrinol (Lausanne). 2014;5:145

22. Ruan HB, Singh JP, Li MD, Wu J, Yang X. Cracking the O-GlcNAc code in metabolism. Trends Endocrinol Metab. 2013;24(6):301-309

23. Li MD, et al. Adipocyte OGT governs diet-induced hyperphagia and obesity. Nat Commun. 2018;9(1):5103.

24. Zachara NE, O’Donnell N, Cheung WD, Mercer JJ, Marth JD, Hart GW. Dynamic O-GlcNAc modification of nucleocytoplasmic proteins in response to stress. A survival response of mammalian cells. J Biol Chem. 2004;279(29):30133-30142.

25. Martinez MR, Dias TB, Natov PS, Zachara NE. Stress-induced O-GlcNAcylation: an adaptive process of injured cells. Biochem Soc Trans. 2017;45(1):237-249.

26. Ngoh GA, Hamid T, Prabhu SD, Jones SP. O-GlcNAc signaling attenuates ER stress-induced cardiomyocyte death. Am J Physiol Heart Circ Physiol. 2009;297(5):H1711-H1719.

27. Yuzwa SA, et al. Increasing O-GlcNAc slows neurodegeneration and stabilizes tau against aggregation. Nat Chem Biol. 2012;8(4):393-399.

28. Banerjee S, et al. Triptolide-induced cell death in pancreatic cancer is mediated by O-GlcNAc modification of transcription factor Sp1. J Biol Chem. 2013;288(47):33927-33938.

29. Wang AC, Jensen EH, Rexach JE, Vinters HV, Hsieh-Wilson LC. Loss of O-GlcNAc glycosylation in forebrain excitatory neurons induces neurodegeneration. Proc Natl Acad Sci USA. 2016;113(52):15120-15125

30. Yang X, et al. Phosphoinositide signalling links O-GlcNAc transferase to insulin resistance. Nature. 2008;451(7181):964-969.

31. Ruan HB, et al. O-GlcNAc transferase/host cell factor $\mathrm{C} 1$ complex regulates gluconeogenesis by modulating PGC-1 $\alpha$ stability. Cell Metab. 2012;16(2):226-237.

32. Li MD, et al. O-GlcNAc signaling entrains the circadian clock by inhibiting BMAL1/CLOCK ubiquitination. Cell Metab. $2013 ; 17(2): 303-310$

33. Ruan HB, et al. Calcium-dependent O-GlcNAc signaling drives liver autophagy in adaptation to starvation. Genes Dev. 2017;31(16):1655-1665

34. Park JK, et al. An endoplasmic reticulum protein, Nogo-B, facilitates alcoholic liver disease through regulation of kupffer cell polarization. Hepatology. 2017;65(5):1720-1734

35. Roychowdhury S, McMullen MR, Pisano SG, Liu X, Nagy LE. Absence of receptor interacting protein kinase 3 prevents ethanol-induced liver injury. Hepatology. 2013;57(5):1773-1783.

36. Pellicoro A, Ramachandran P, Iredale JP, Fallowfield JA. Liver fibrosis and repair: immune regulation of wound healing in a solid organ. Nat Rev Immunol. 2014;14(3):181-194.

37. Tsuchida T, Friedman SL. Mechanisms of hepatic stellate cell activation. Nat Rev Gastroenterol Hepatol. 2017;14(7):397-411.

38. Marshall KD, Baines CP. Necroptosis: is there a role for mitochondria? Front Physiol. 2014;5:323.

39. Seo J, et al. CHIP controls necroptosis through ubiquitylation- and lysosome-dependent degradation of RIPK3. Nat Cell Biol. 2016;18(3):291-302.

40. He S, et al. Receptor interacting protein kinase-3 determines cellular necrotic response to TNF-alpha. Cell. 2009;137(6):1100-1111.

41. McGreal SR, et al. Modulation of O-GlcNAc Levels in the Liver Impacts Acetaminophen-Induced Liver Injury by Affecting Protein Adduct Formation and Glutathione Synthesis. Toxicol Sci. 2018;162(2):599-610.

42. Cho YS, et al. Phosphorylation-driven assembly of the RIP1-RIP3 complex regulates programmed necrosis and virus-induced inflammation. Cell. 2009;137(6):1112-1123.

43. Guicciardi ME, Malhi H, Mott JL, Gores GJ. Apoptosis and necrosis in the liver. Compr Physiol. 2013;3(2):977-1010.

44. Vucur M, et al. RIP3 inhibits inflammatory hepatocarcinogenesis but promotes cholestasis by controlling caspase-8- and JNK-dependent compensatory cell proliferation. Cell Rep. 2013;4(4):776-790.

45. Xu W, et al. O-GlcNAc transferase promotes fatty liver-associated liver cancer through inducing palmitic acid and activating endoplasmic reticulum stress. J Hepatol. 2017;67(2):310-320. 
46. Mauad TH, et al. Mice with homozygous disruption of the mdr2 P-glycoprotein gene. A novel animal model for studies of nonsuppurative inflammatory cholangitis and hepatocarcinogenesis. Am J Pathol. 1994;145(5):1237-1245.

47. Wakabayashi K, et al. IL-2 receptor alpha(-/-) mice and the development of primary biliary cirrhosis. Hepatology. 2006;44(5):1240-1249.

48. Oertelt S, et al. Anti-mitochondrial antibodies and primary biliary cirrhosis in TGF-beta receptor II dominant-negative mice. J Immunol. 2006;177(3):1655-1660.

49. Shafi R, et al. The O-GlcNAc transferase gene resides on the $\mathrm{X}$ chromosome and is essential for embryonic stem cell viability and mouse ontogeny. Proc Natl Acad Sci USA. 2000;97(11):5735-5739.

50. Song Y, et al. H19 promotes cholestatic liver fibrosis by preventing ZEB1-mediated inhibition of epithelial cell adhesion molecule. Hepatology. 2017;66(4):1183-1196. 